\title{
Training Residents in Community Health Centers: Facilitators and Barriers
}

\author{
Carl G. Morris, MD, MPH \\ Frederick $M$. Chen, $M D, M P H^{2}$ \\ ${ }^{\prime}$ Group Health Cooperative Family Medi- \\ cine Residency, Department of Family Med- \\ icine, University of Washington, Seattle, \\ Washington \\ ${ }^{2}$ Department of Family Medicine, Univer- \\ sity of Washington School of Medicine, \\ Seattle, Washington
}

\begin{abstract}
PURPOSE Training family medicine residents in underserved settings, such as community health centers ( $\mathrm{CHCs}$ ), may provide a solution to the primary care workforce shortage. We sought to describe the facilitators and barriers to creating partnerships between CHCs and family medicine residencies (FMRs).
\end{abstract}

METHODS We conducted 19 key informant interviews and 3 focus groups to identify the key factors in the CHC-FMR relationship. Audiotapes and transcripts were analyzed to identify major themes. Key informant results were validated and expanded in the focus group discussions.

RESULTS Four major themes describe the CHC-FMR training partnership: mission, money, quality, and administrative/governance complexity. The CHC-FMR training affiliation is a complex relationship drawn together by a shared mission of service to the underserved, enhanced financial stability, workforce improvement, and greater educational and clinical quality. The relationship is hindered by competing primary missions, chronic underfunding, complex governing institutional regulations, and administrative challenges. In addition, the focus groups offered several policy solutions to address the barriers to CHC-FMR affiliation.

CONCLUSIONS A successful CHC-FMR training partnership relies upon the development of a shared mission of education and service, as well as innovation and flexibility by the organizations that govern them.

Ann Fam Med 2009;7:488-494. doi:10.1370/afm.1041.

\section{INTRODUCTION}

$\mathrm{F}$ amily medicine residencies (FMRs) and Community Health Centers - (CHCs) are confronted with 2 different aspects of the current primary care workforce crisis in the United States. ${ }^{1.7}$ FMRs suffer from declining student interest in primary care and perennial threats to financial solvency. ${ }^{7-10}$ During the past decade there has been a $52.6 \%$ decrease in US medical school graduates choosing careers in family medicine. ${ }^{11}$ Similarly, the increasing number of FMR closures has led to a decrease in the number of FMR positions offered. ${ }^{11,12}$ The financial viability of existing FMRs has been further threatened by cuts in federal programs designed to train primary care physicians, such as funding of graduate medical education through the Medicare program and Title VII programs. ${ }^{11-13}$

$\mathrm{CHCs}$ are primary care clinics that serve uninsured and underserved populations. Also known as Federally-Qualified Health Centers, their difficulties in recruiting physicians are well-documented. ${ }^{14-18}$ A 2004 national survey of $890 \mathrm{CHCs}$ found serious shortages of physicians, especially family physicians, who make up the primary physician workforce of CHCs. ${ }^{19}$ Since this survey, CHCs have expanded their services from 8 million patients per year at 3,300 delivery sites to 18 million patients and 6,600 delivery sites. ${ }^{20-22}$ This expansion required a $57 \%$ increase in the number of primary care physicians working in $\mathrm{CHCs}_{1}{ }^{23}$ exacerbating the already chronic problems of recruitment and retention of health care clinicians 
in underserved areas ${ }^{14,16,24}$ and the shortage of family physicians. ${ }^{25-30}$

Our current workforce crisis threatens the health care safety net system and the vulnerable population it serves. The doubling of CHC services during the Bush administration improved access to care for the underserved ${ }^{31}$; however, the success of the $\mathrm{CHC}$ expansion is challenged by the decrease in the number of physicians choosing careers in primary care in general and electing to work in underserved areas specifically. Furthermore, by 2015 the CHC's Access for All America initiative plans for another doubling of patients served, ${ }^{32}$ which will require a corresponding increase in the primary care workforce. Investment in the health care workforce pipeline is essential to meet these workforce demands.

One potential solution for both FMRs and CHCs is a CHC-FMR affiliation in which a FMR clinic is located in and partners with a CHC. Such affiliations have existed for more than 20 years; they provide effective training and increase the number of graduates working in safety net health care settings. ${ }^{33-38}$ Graduates of these programs are 4 times more likely than graduates from nonaffiliated programs to continue to work in CHCs. A recent analysis from the WWAMI (Washington, Wyoming, Alaska, Montana, Idaho) Family Medicine Residency Network showed that $80 \%$ of graduates from residency programs affiliated with $\mathrm{CHC}$ sorked in underserved areas in the year after graduation. ${ }^{33}$

That the number of CHC-FMR residency programs has remained at approximately 25 to 30 for the past 20 years suggests barriers to affiliation have limited expansion of these programs. ${ }^{39,40}$ Historically, motivation by both parties to train residents in $\mathrm{CHC}$ s has been strong. Successful affiliations have resulted in improved physician recruitment to underserved areas, high residenttraining satisfaction, improved quality of care within $\mathrm{CHCs}$, improved physician-training environments, and enhanced FMR clinical revenue. ${ }^{34-39,43-46}$ CHC-FMR affiliations have failed because they lacked a shared mission of education and service, ${ }^{44}$ and because they were beset with the challenges of chronic financial instability ${ }^{39,41,42}$ and administrative and governance complexity. ${ }^{39,44,45}$

The literature on CHC-FMR affiliation is limited to descriptions of individual programs, articles that address issues of financing or workforce, and qualitative reports. ${ }^{34-39,41-46}$ There have been no structured qualitative analyses of the CHC-FMR affiliations, and published evidence is insufficient to inform CHCs and FMRs in the development of successful partnerships. Accordingly, in the autumn of 2004 the WWAMI Family Medicine Residency Network created a work- ing group to identify the barriers to and facilitators of CHC-FMR affiliations in their region and to determine ways to enhance future partnerships.

\section{METHODS}

Using key informant interviews and focus groups, we conducted a qualitative study of the CHC-FMR affiliations in the 17-residency WWAMI Family Medicine Residency Network. CHC-FMRs were defined as residencies in which the primary outpatient continuity clinic training occurs in a CHC. During the first stage, key informant interviews were analyzed to create a thematic framework of facilitators and barriers. During the second stage, this proposed framework was tested in focus groups of CHC and FMR leaders and further refined. The University of Washington Institutional Review Board approved this study.

\section{Key Informant Interviews}

Key informants were selected based on their experience with and knowledge of CHC-FMR affiliations. Their programs, some of which had been in existence for 20 years, represented the full spectrum of experience and success throughout the WWAMI residency network: 2 affiliations had failed, 6 programs had been affiliated for longer than 3 years, and 1 new $\mathrm{CHC}$ FMR program had previously failed and later reestablished an affiliation. There were 2 models of affiliation: 1 included 4 satellite CHC-FMR programs with 2 residents in each residency year (the main residency site was not $\mathrm{CHC}$-affiliated); and 1 included 4 CHC-FMR programs in which the main FMR training clinic was a CHC with 8 to 10 residents in each residency year. Purposive sampling yielded 19 key informants distributed across 4 states. Informants included $\mathrm{CHC}$ administrators, residency directors, hospital administrators, and board members.

A structured interview tool was developed, pilottested, and modified to identify the key components of the barriers to and facilitators of the CHC-FMR collaboration. One author (C.M.) conducted all interviews. Interviews lasted 1 hour and were audiorecorded and transcribed. The transcribed interviews were read, coded, and thematically labeled independently by 2 investigators (C.M., F.C.) using qualitative analysis software (ATLAS-ti 5.0, ATLAS-ti, GmbH, Berlin, Germany). ATLAS-ti has an open-coding technique $^{47}$ that allows coders to organize and evaluate similar quotations by themes. Codes were then collated and combined into a thematic framework, and differences between codes were reconciled through mutual agreement. We did not compare or group responses by size, type, or success of CHC-FMR affiliation. 


\section{Focus Groups}

In the second stage of the study, we conducted 3 focus groups to validate the facilitators of and barriers to CHC-FMR partnerships identified during the key informant interviews, and to identify potential ways to address the barriers. Attendees were selected to represent both CHCs and FMRs across a 10-state region (Alaska, Washington, Oregon, Montana, Idaho, Wyoming, Utah, Colorado, North Dakota, and South Dakota), to include representatives from other CHCFMR programs, and to expand the generalizability of the study's findings. Focus group participants were chosen for their knowledge of, experience with, and interest in CHC-FMR affiliations. The 25 attendees included $10 \mathrm{CHC}$ administrators, $5 \mathrm{CHC}$ medical directors, 7 FMR directors, 1 CHC board member, 4 primary care association administrators, 2 university faculty members, 1 independent consultant, and 1 state government representative.

The focus group sessions lasted 2.5 hours, were facilitated by the regional primary care association executive directors and the study authors, and were audio-recorded. One study member was also present to organize the discussion and document it by taking notes. Each focus group followed a standard outline that began with a review of themes derived from the key informant interviews. We created summaries of the 3 focus groups by reviewing the audiotapes and combining the notes from the facilitators and study members who documented the sessions. Summaries of each focus group and the combined analysis of all focus groups were circulated to the focus group participants for their comments. As with the key informant interviews, the transcripts of the focus groups were read and coded. These codes were reconciled between both investigators and used to revise the thematic framework.

\section{RESULTS}

Analysis of the key informant interviews and focus groups yielded 4 themes that describe the barriers to and facilitators of CHC-FMR affiliations: (1) mission, (2) money, (3) governing institutional barriers and administrative challenges, and (4) quality. Mission and money present both barriers to and facilitators of CHC-FMC relationships. The governing institutional requirements of each organization $(\mathrm{CHC}$ and FMR) and the administrative challenges of meeting multiple governance requirements and administering a combined clinical and teaching enterprise hinder these relationships. Enhanced clinical and educational quality that comes from the collaboration between a service and academic partnership promotes successful partnerships.
Additionally, focus groups identified 3 ways to overcome barriers to successful affiliation: (1) a shared mission and vision of service and education; (2) new reimbursement streams that facilitate the shared mission, do not threaten existing funding streams, and account for the costs of outpatient training; and (3) clear communication of governance requirements and administrative roles.

\section{Mission}

The key informants and focus groups identified mission as centrally important to the affiliation. Participants believed strongly that the successful CHC-FMR programs identified commonalities and differences in the mission and culture of each organization and actively worked to combine them. Both CHCs and FMRs share a mission for service to the underserved and their communities. One FMR director pointed out the common missions of service:

We've taken care of the underserved. We are the safety nets in our communities.... We both have the same purpose (FMR director 1).

The mission of service is primary to CHCs, but it is secondary to the mission of education for FMRs. Problems related to finance, governance, or administration often sharpened the differences of mission between CHCs and FMRs and created barriers to successful partnership.

If you're a residency-based $\mathrm{HC}$ [health center], the residency culture is going to dominate because that's your fundamental purpose. If you're a HC-based residency, then you expect that the HC culture would dominate, but clearly there's got to be a mixture (CHC Administrator 2).

The key informant and focus group attendees believed strongly that the most successful affiliations constructed a joint mission and vision statement, communicated this statement clearly, and followed it throughout all levels of both organizations. This joint statement served as a charter for decision making.

\section{Money}

Financial issues also facilitated and created barriers to CHC-FMR affiliation. Although participants acknowledged that financial issues are not unique to CHCFMRs, chronic underfunding of both organizations creates a culture in which both are highly motivated to increase funding and aggressively protect existing funding streams.

Participants frequently commented that CHCs and FMRs are attracted to the improved financial stability resulting from collaboration, but each is limited in its ability to absorb the costs of affiliation. Participants 
described how affiliation is facilitated by improved reimbursement (such as $\mathrm{CHC}$ cost-based reimbursement for Medicaid and Medicare patients), cost-savings resulting from improved recruitment of physicians and residents, and Federal Tort Claims Act liability coverage through the CHC. For example, one FMR director reported:

It's a very inexpensive way to train residents because you don't have the expensive clinic and clinic staff, and you still get the Medicare GME [graduate medical education], which if it's big enough, it works out well for you, too (FMR director 4).

In another example, a hospital administrator reported the improvement in physician retention:

Without question, since those community health centers became training sites, the physician population is stabilizing. There used to be huge turnover in the docs who worked there (Hospital administrator 5).

Conversely, economic factors also presented barriers to affiliation. Chronic underfunding of CHCs and FMRs results in each organization wanting to protect its respective funding streams and limits its ability to absorb costs not associated with its primary mission. Scarce resources further affect the trust and partnership necessary to form effective affiliations. There are considerable costs associated with affiliation that are not reimbursed, such as increased administrative requirements of residency training, increased direct and indirect clinical costs, and decreased productivity. Participants from both CHCs and FMRs agreed that the indirect costs of providing residency training are not adequately accounted for in the current graduate medical education reimbursement model. They further commented that $\mathrm{CHC}$ s do not have a $\mathrm{CHC}$-specific mechanism to account for the costs of providing training and noted the shortsightedness of not investing in health workforce training. As a CHC administrator summarized:

I think so much of the tension is all about money. If we could get the money issue resolved, I think a lot of the other conflicts would dissolve if we weren't always, both groups, worrying about money ( $\mathrm{CHC}$ administrator 1$)$.

\section{Administrative and Governance Complexity}

In addition to barriers generated by aspects of mission and money, the study respondents identified the number of governing bodies and complexity of meeting the governance and administrative requirements necessary to run a FMR and a CHC as serious barriers to successful affiliation: as barriers to initiating the relationship, as ongoing challenges to maintain it, and frequently as major causes of failure of the affili- ation. Both organizations are governed by different rules, and successful affiliation requires compliance with both of the governing bodies' regulations. One FMR administrator listed the following organizations involved in the governance of the CHC-FMR affiliation:

[T] he ACGME [American Council for Graduate Medical Education], RRC [Residency Review Committee], Accreditation Association of Ambulatory Health Care, Joint Commission on Accreditation of Healthcare Organization, the Health Resources and Services Administration, Centers for Medicare and Medicaid Services, sponsoring hospitals, $\mathrm{CHC}$ administration, $\mathrm{CHC}$ boards, FMR administration, and university administration (FMR administrator 1).

A common complaint was that the lack of flexibility for innovation by organizations involved in the governance of the affiliations hindered program development. One CHC administrator illustrated this well when she stated:

...part of the problems that we had with coming up with an affiliation agreement was because everything we tried to propose, that was kind of a little bit more straightforward and civil, was against a government regulation (CHC administrator 3).

In addition, the redundancy in administrative structure, mandated by governing bodies and inherent in running both a CHC and a FMR, was considered a major barrier to successful affiliation. Participants commented that with 2 administrative structures, the issues of communication, scheduling, conflicting roles and responsibilities, all served as barriers to effective collaboration. Specific examples of conflict and difficulty include the discontinuity of residency clinic functions, increased staffing needs, challenges to the provision of continuity of patient care, and the balance of clinic needs with those of the residency. Further, cycles of credentialing, obtaining privileges, maintaining oversight, conducting orientations, interviewing new residents, and introducing new physicians into the practice associated with FMRs bring with them administrative responsibilities not usually associated with CHCs.

Operating as a $\mathrm{CHC}$ changes our lives immensely in terms of keeping the mission in front of everybody, keeping abreast of all the regulatory requirements for residency education and health centers, and keeping in compliance with all of those things (FMR director 3).

\section{Quality}

In addition to the shared mission and financial benefits, participants remarked that the CHC-FMR partner- 
ship, which blended the academic influence of the residency with $\mathrm{CHC}$ standards for clinical care of the underserved, greatly improved the quality of the residents' education and the patients' medical care. Study participants believed the FMR training benefits from the community-based training in underserved populations, culturally appropriate training, and role modeling from $\mathrm{CHC}$ physicians; and the $\mathrm{CHC}$ benefits from the recruitment of high-quality residents and faculty. One FMR director described the effect of their affiliation with a $\mathrm{CHC}$ :

We see a heightened level of service and patient care because residents are learning and somebody is mentoring them, and so the consequences of that are the underserved are treated and served at a higher level than what you would find at a regular community health center (FMR director 5).

There were other aspects of quality improvement reported by participants. FMR representatives reported a marked increase in medical student interest after an affiliation with a CHC. Also, clinical care was believed to be improved by the combination of chronic disease management infrastructure of $\mathrm{CHCs}$, expanded scope of CHC services (pharmacy, social work, mental health, etc), and evidence-based medicine from the FMR.

Being involved with a community health center, you understand the dilemma of the underserved, the magnitude of the problem, the importance of the personal satisfaction of taking care of the underserved. And the residents gain from that (CHC administrator 1).

\section{Policy Solutions}

The focus groups were asked to brainstorm ways to overcome barriers to affiliation. They identified 3 solutions for overcoming the barriers: (1) a shared mission and vision of service and education for $\mathrm{CHC}$ $\mathrm{FMRs}_{\text {; }}$ (2) new reimbursement streams that facilitate the shared mission, do not threaten existing funding streams, and account for the costs of outpatient training; and (3) clear communication of governance requirements and administrative roles.

\section{Shared Mission of Service and Education} All focus groups agreed that the mission should be a shared vision of service and education to avoid creating separate mission silos for CHCs and FMRs. The following recommendations regarding mission were proposed: (1) the CHC-FMR affiliation must have a blended vision of service and education; (2) the vision statement should reflect a mission of service and workforce supply for underserved populations; (3) the shared mission must be communicated throughout the entire organization; (4) funding should be realigned so that reimbursement facilitates the shared mission of service and education; (5) and the mission should contain the long-range vision of health workforce supply and quality of care. The success of the model depends on a paradigm shift from thinking of the model from the perspective of either the FMR or the CHC to the perspective of an organization that shares an equal mission of service and education.

\section{New Reimbursement Streams}

Regarding financing, the focus groups proposed that (1) funding should be based on the success of the shared mission of service and education; (2) reimbursement should motivate and facilitate the shared mission; (3) reimbursement should be calculated separately from comparisons with other entities that do not provide clinical care and residency education; (4) funding should not challenge funding streams for nonaffiliated CHCs and FMRs (Public Health Services Act 330 grants for $\mathrm{CHC}$ or graduate medical education reimbursement for FMRs); and (5) reimbursement needs to account for outpatient training.

Delineation of Governance and Administrative Roles The focus groups also agreed that the governance and administrative roles must be explicit. Participants believed that the roles and responsibilities of residency program director should be specifically defined. Currently the residency director reports to the Accreditation Council of Graduate Medical Education, the Residency Review Committee, and the sponsoring hospital. The director also needs to connect with the CHC leadership to allow for flexibility in sharing a joint mission. Similarly, the clinic manager and medical director roles must be defined in a way that allows them to work within the governance limitations of both the $\mathrm{CHC}$ and the FMR. These positions should be balanced structurally so that the dual responsibilities of clinic operations and education are reconciled.

It was noted that the $\mathrm{CHC}$ community board requirement (at least $51 \%$ of the board members must be patients in the $\mathrm{CHC}$ ) should not be changed in a CHC-FMR affiliation. Other recommendations included (1) an overlap and understanding of the requirements of each other's governing bodies; (2) a new funding line that supports shared governance, and a blended mission that directs funds to graduate medical education that serves patients in $\mathrm{CHCs}_{\text {; }}$ and (3) demonstration projects to facilitate innovations that require a relaxation of the regulatory bodies' standards.

\section{DISCUSSION}

This study shows that the CHC-FMR affiliation is a complex relationship in which both organizations 
are drawn together by the shared mission of service to the underserved, the need for improved financial stability, and the opportunity to improve clinical and educational quality. Both organizations benefit from improved workforce recruitment, enhanced patient care, and an improved teaching environment. The affiliation, however, is hindered by competing organizational missions, chronic underfunding, governing institutional regulations, and administrative challenges. A successful CHC-FMR affiliation relies on its ability to develop a shared mission of education and service, as well as innovation and flexibility by the organizations that govern them.

The growth in the number of CHCs and patients served, combined with a decrease in the number of primary care trainees, has exacerbated a health workforce crisis and necessitated changes in medical education policy. ${ }^{1-7,28-30} \mathrm{CHC}$-FMR affiliations offer one possible solution to this workforce shortage through enhanced teaching and recruitment of residents to underserved areas, $^{33-40,44}$ This study provides a description of the factors that determine a successful affiliation between CHCs and FMRs, and adds to the call for adoption of policies to pave the way for more successful partnerships. ${ }^{6,16,17,23,32,48-51}$ We found 4 themes that describe the facilitators of and barriers to CHC-FMR affiliations: (1) mission, (2) money, (3) governing institutional barriers and administrative challenges, and (4) quality.

Our study has several limitations. Bias introduced by the investigators or by the selection of participants is a potential weakness of any qualitative study. We attempted to limit this bias by using standardized interview tools, having 2 independent coders, validating the thematic analysis of the key informant interviews with focus groups, and selecting participants with both regional and institutional representation. Regional variability may also limit the applicability of these findings.

Policy solutions can facilitate improved workforce training for the underserved through CHC-FMR affiliations by addressing the governance and financing barriers that hinder affiliation. Aligning reimbursement with clinical production and education, directly linking graduate medical educational funding to ambulatory care training sites based on outpatient training costs, raising the cap on graduate medical education-supported ambulatory training positions, and developing cost-based reimbursement for educational costs in Medicaid and Medicare reimbursement are all changes that would enhance and support the growth of $\mathrm{CHC}$ FMR affiliations.

Policy makers should be aware of the improvements in quality of care and the health workforce benefits, as well as the challenges of training family medicine resi- dents in CHCs. Graduates of CHC-FMR affiliations are approximately twice as likely to work in safety net settings after graduation. ${ }^{33}$ Changes in governance and financing will be necessary, however, to avoid being barriers to growth adequate to meet the workforce needs of safety net settings. A health center administrator with years of CHC-FMR experience said it well: "I still believe this is a match made in heaven. It's a little rocky path to heaven sometimes."

To read or post commentaries in response to this article, see it online at http://www.annfammed.org/cgi/content/full/7/6/488.

Key words: Physicians, family/manpower; medically underserved area; medical residency; community health centers

Submitted April 2, 2008; submitted, revised, June 2, 2009; accepted June 12, 2009.

Presented as "The Community Health Center-Family Medicine Residency Affiliation: A Qualitative Analysis," at the 40th Society of Family Medicine Annual Spring Conference, April 2007, Chicago, Illinois.

\section{References}

1. The impending collapse of primary care medicine and its implications for the state of the nation's health care. Sep 21, 2006. http:// www.acponline.org/hpp/statehc06_1.pdf. Accessed Oct 25, 2006.

2. Brotherton SE, Rockey PH, Etzel SI. US graduate medical education, 2004-2005: trends in primary care specialties. JAMA. 2005;294(9): 1075-1082.

3. Garibaldi RA, Popkave C, Bylsma W. Career plans for trainees in internal medicine residency programs. Acad Med. 2005;80(5): 507-512.

4. Whitcomb ME. Primary care medicine in the United States: where are we headed? Acad Med. 2002;77(8):759-760.

5. Whitcomb ME, Cohen JJ. The future of primary care medicine. N Engl J Med. 2004;351(7):710-712.

6. Council on Graduate Medical Education. Tenth report: Physician Distribution and Health Care Challenges in Rural and Inner-City Areas. http://www.cogme.gov/rpt10.htm.

7. The US Primary Care Physician Workforce. Minimal Growth, 19801999. WwW.graham-center.org/x467.xml.

8. Pugno PA, McGaha AL, Schmittling GT, Fetter GT Jr, Kahn NB Jr. Results of the 2006 national resident matching program: family medicine. Fam Med. 2006;38(9):637-646.

9. McPherson DS, Schmittling GT, Pugno PA, Kahn NB Jr. Entry of US medical school graduates into family medicine residencies: 2003 2004 and 3-year summary. Fam Med. 2004;36(8):553-561.

10. Hueston WJ, Mainous AG III, Ye X. The financial status of departments of family medicine at US medical schools. Fam Med. 2001;33(3):166-170.

11. Pugno PA, McGaha AL, Schmittling GT, DeVilbiss A, Kahn NB Jr. Results of the 2007 national resident matching program: family medicine. Fam Med. 2007;39(8):562-571.

12. Gonzalez EH, Phillips RL Jr, Pugno PA. A study of closure of family practice residency programs. Fam Med. 2003;35(10):706-710.

13. Family Physician Workforce Reform as approved by the 2006 Congress of Delegates. Recommendations of the American Academy of Family Physicians. http://www.aafp.org/workforce. Accessed Jul 13, 2007. 
14. Singer JD, Davidson SM, Graham S, Davidson HS. Physician retention in community and migrant health centers: who stays and for how long? Med Care. 1998;36(8):1198-1213.

15. Alpert JJ. Serving the medically underserved. Am J Public Health. 1998;88(3):347-348.

16. Politzer RM, Harris DL, Gaston MH, Mullan F. Primary care physician supply and the medically underserved. A status report and recommendations. JAMA. 1991;266(1):104-109.

17. Salsberg ES, Forte GJ. Trends in the physician workforce, 1980 2000. [Milwood]. Health Aff (Millwood). 2002;21(5):165-173.

18. Bowman RC. Birth origins and FP choice. http://www.unmc.edu/ Community/ruralmeded/birth_origins_ethnicity_FP.htm. Accessed Dec 4, 2008.

19. Rosenblatt RA, Andrilla CH, Curtin T, Hart LG. Shortages of medical personnel at community health centers: implications for planned expansion. JAMA. 2006;295(9):1042-1049.

20. Landa A. Community Health Centers: Casting a wider safety net. amednews.com. Sept 2; 1-6. http://www.ama-assn.org/amednews/ 2002/09/02/gvsa0902.htm.

21. National Association of Community Health Centers. 2004-2005 Annual Report: National Association of Community Health Centers. 2005.

22. America's Health Centers: Fact Sheet: National Association of Community Health Centers; August 2008.

23. Access Transformed. Building a primary care workforce for the $21^{\text {st }}$ century. http://www.nachc.com/client/documents/ACCESS\%20Transf ormed\%20full\%20report.PDF, Aug 2008.

24. Health Resources and Services Administration. HRSA's long range action plan: report on access to primary care for all. 1990.

25. O'Malley AS, Forrest CB, Politzer RM, Wulu JT, Shi L. Health center trends, 1994-2001: what do they portend for the federal growth initiative? Health Aff (Millwood). 2005;24(2):465-472.

26. Shekar S. Update from the Bureau of Primary Health Care to the National Association of Community Health Centers. Paper presented at: Remarks to the National Association of Community Health Centers; March 24, 2003.

27. Green LA DM, Ruddy G. The Physician Workforce of the United States: A Family Medicine Perspective. Washington DC: The Graham Center; 2004.

28. Health Resources and Services Administration. Physician Supply and Demand: Projections to 2020 October 2006.

29. Council On Graduate Medical Education. Physician workforce Policy Guidelines for the U.S. for 2000-2020. Rockville, MD: US Department of Health and Human Services; 2005.

30. American Association. of Medical Colleges Recent Studies and Reports on Physician Shortages in the U.S. Center for Workforce Studies; 2007.

31. Sack K. Expansion of clinics shapes bush legacy. New York Times, December 25, 2008. http://www.nytimes.com/2008/12/26/health/ policy/26clinics.html. Accessed Feb 9, 2009.

32. Access For All America Plan. http://www.nachc.org/aaa-docs.cfm. March 2008. Accessed Feb 9, 2009.
33. Morris CG, Johnson D, Kim S, Chen F. Training family medicine residents in community health centers: a health workforce solution. Fam Med. 2008;40(4):271-276.

34. Engebretsen BJ. Family medicine and community health centers: a natural alliance. Fam Med. 1989;21(6):417-418.

35. Cooksey JA, Kaur K, Matters MD, Simone B, Chun E, Hoekstra A Community health centers: unrecognized partners in health professions education. J Health Care Poor Underserved. 1999;10(3):349-361.

36. Hill L, Patrick K, Avila P. Training physicians to care for the underserved: preventive medicine residency-community health center linkages. Am J Prev Med. 1996;12(3):156-160.

37. Tallia AF, Micek-Galinat L, Formica PE. Academic-community linkages: community-based training for family physicians. Fam Med. 1996;28(9):618-623.

38. Gordon PR, Hale F. The service-education linkage: implications for family practice residency programs and community and migrant health centers. Fam Med. 1993;25(5):316-321.

39. Hale FA GP, Denton D, Warrick LH. The Family Practice Residency Program Community/Migrant Health Center Linkage Manual; 1993.

40. Morris CG, Chen FM, Andrilla CH. Training Family Physicians in Community Health Centers: A National Survey. 2008 . In press.

41. Jones TF. The cost of outpatient training of residents in a community health center. Fam Med. 1997;29(5):347-352.

42. Jones TF, Culpepper $L$, Shea $C$. Analysis of the cost of training residents in a community health center. Acad Med. 1995;70(6):523-531.

43. Prislin MD, Morohashi D, Dinh T, Sandoval J, Shimazu H. The community health center and family practice residency training. Fam Med. 1996;28(9):624-628.

44. Zweifler J. Balancing service and education: linking community health centers and family practice residency programs. Fam Med. 1993;25(5):306-311.

45. Redington TJ, Lippincott J, Lindsay D, Wones R. How an academic health center and a community health center found common ground. Acad Med. 1995;70(1):21-26.

46. Zweifler J. Family practice residencies in community health centers-an approach to cost and access concerns. Public Health Rep. 1995;110(3):312-318

47. Kennedy TJ, Lingard LA. Making sense of grounded theory in medical education. Med Educ. 2006;40(2):101-108.

48. Grumbach K, Hart LG, Mertz E, Coffman J, Palazzo L. Who is caring for the underserved? A comparison of primary care physicians and nonphysician clinicians in California and Washington. Ann Fam Med. 2003;1(2):97-104.

49. Tavernier LA, Connor PD, Gates D, Wan JY. Does exposure to medically underserved areas during training influence eventual choice of practice location? Med Educ. 2003;37(4):299-304.

50. Council of Graduate Medical Education. Improving access to health care through physician workforce reform: Directions for the 21st century. Third report of the Council of Graduate Medical Education (DHHS Pub. No. [PHS]). 1992.

51. Pathman DE. Medical education and physicians' career choices: are we taking credit beyond our due? Acad Med. 1996;71(9):963-968. 